\begin{tabular}{|c|c|c|}
\hline & $\begin{array}{c}\text { Gazi University } \\
\text { Journal of Science }\end{array}$ & 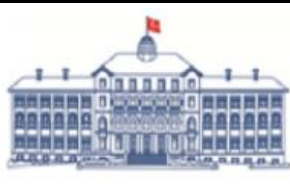 \\
\hline & http://dergipark.gov.tr/gujs & \\
\hline
\end{tabular}

\title{
On the Hankel Determinant of $\boldsymbol{m}$-fold Symmetric Bi-Univalent Functions Using a New Operator
}

\author{
Waggas Galib ATSHAN ${ }^{1}$ (D) , Reaam Abd AL-SAJJAD ${ }^{1}$ (D) Sahsene ALTINKAYA $^{2, *(D)}$ \\ ${ }^{1}$ University of Al-Qadisiyah, College of Science, Diwaniyah, Iraq \\ ${ }^{2}$ Beykent University, Faculty of Arts and Sciences, 34500, Istanbul, Turkey
}

\section{Highlights}

- This paper focuses on definition of a new subclass of bi-univalent functions.

- The convolution is proposed for construction of the operator $\mathcal{J}_{s, a, \mu}^{\delta, \lambda} f(z)$.

- The second Hankel determinant were obtained.

\section{Article Info}

Received: 06 July 2021 Accepted: 24 Jan 2022

\section{Keywords}

Univalent function

Coefficient estimate

Hankel determinant

\begin{abstract}
In this article, we aim to describe a new operator $\mathcal{J}_{s, a, \mu}^{\delta, \lambda}$ via convolution. Moreover, we aim to present a new subclass $\boldsymbol{C}_{\Sigma \mathrm{m}}(\tau ; \beta)$ related to $m$-fold symmetric bi-univalent functions in the open unit disk $\Theta=\{\mathrm{z} \in \mathbb{C}:|\mathrm{z}|<1\}$. Finally, an estimate related to the Hankel determinant for functions in $\boldsymbol{C}_{\Sigma \mathrm{m}}(\tau ; \beta)$ are given.
\end{abstract}

\section{INTRODUCTION}

Assume $\mathcal{A}$ is the class of normalized analytic functions in $\Theta$ with Taylor series

$f(z)=z+\sum_{k=2}^{\infty} a_{k} z^{k}$

Assume $\mathcal{S}$ is the class of univalent functions from $\mathcal{A}$ in $\Theta$. Further, every function $f \in \mathcal{S}$ has an inverse $f^{-1}$ fulfilling $f^{-1}(f(z))=z(z \in \Theta)$ and $f\left(f^{-1}(w)\right)=w\left(w \in \Theta_{p}\right)$, where $p \geq \frac{1}{4}$ denotes the radius of the image $f(\Theta)$ and $\Theta p=\{z \in \mathbb{C}:|z|<p\}$ [1]. It is recalled that

$g(w)=f^{-1}(w)=w+C_{2} w^{2}+C_{3} w^{3}+C_{4} w^{4}+\cdots$,

where

$C_{2}=-a_{2}$

$C_{3}=\left(2 a_{2}^{2}-a_{3}\right)$

$C_{4}=-\left(5 a_{2}^{3}-5 a_{2} a_{3}+a_{4}\right)$.

A function $f \in \mathcal{A}$ is named bi-univalent in $\Theta$ if both $f$ and $f^{-1}$ are univalent in $\Theta$. Next, assume $\sum$ is the class of bi-univalent functions $f \in \mathcal{A}$ in $\Theta$. For a detailed literature and fundamental examples of $\sum$, see the leading paper by Srivastava et al. [2] (see also [3-10]). 
Assume $f \in \mathcal{A}$ and $g$ is given by $g(z)=z+\sum_{k=2}^{\infty} b_{k} z^{k}$. The convolution (Hadamard product) of $f$ and $g$ is represented by $(f * g)(z)$ and expressed by

$(f * g)(z)=z+\sum_{k=2}^{\infty} a_{k} b_{k} z^{k}$

Aldweby and Darus [11] established the Ruscheweyh type $q$-analogue operator $\mathcal{R}_{q}^{\delta}$ by

$\mathcal{R}_{q}^{\delta} f(z)=z+\sum_{k=2}^{\infty} \frac{[k+\delta-1]_{q} !}{[\delta]_{q} ![k-1]_{q} !} a_{k} z^{k}$

where $\delta \geq 0$. Also, as $q \rightarrow 1^{-}$, we have

$$
\begin{aligned}
\lim _{q \rightarrow 1^{-}} \mathcal{R}_{q}^{\delta} f(z)= & z+\lim _{q \rightarrow 1^{-}}\left[\sum_{k=2}^{\infty} \frac{[k+\delta-1]_{q} !}{[\delta]_{q} ![k-1]_{q} !} a_{k} z^{k}\right] \\
& =z+\sum_{k=2}^{\infty} \frac{(k+\delta-1) !}{(\delta) !(k-1) !} a_{k} z^{k} \\
& =\mathcal{R}_{q}^{\delta} f(z)
\end{aligned}
$$

Komatu [12] introduced a family of integral operator $J_{\mu}^{\lambda}: \Sigma \rightarrow \Sigma$ by

$J_{\mu}^{\lambda} f(z)=z+\sum_{k=1}^{\infty}\left(\frac{\mu}{\mu+k-1}\right)^{\lambda} a_{k} z^{k}, \quad\left(z \in U^{*}=\Theta \backslash\{0\}, k>1, \lambda \geq 0\right)$.

By using the Hurwitz - Lerch Zeta function

$\emptyset(z, s, a)=\sum_{k=0}^{\infty} \frac{z^{k}}{(k+a)^{s}}\left(a \in \mathbb{R} / z_{0}^{-}, s \in \mathbb{R}\right.$ when $\left.0<|z|<1\right)$

and $G_{s, a(z)}$ is given by

$G_{s, a(z)}=(1+a)^{s}\left[\emptyset(z, s, a)-a^{-s}\right]$,

the linear operator $I_{s, a, \mu}^{\lambda} f(z): \Sigma \rightarrow \Sigma$ is expressed by [13]

$I_{s, a, \mu}^{\lambda} f(z)=G_{s, a(z)} * J_{\mu}^{\lambda} f(z)=z+\sum_{k=2}^{\infty}\left(\frac{1+a}{t+a}\right)^{s}\left(\frac{\mu}{\mu+k-1}\right)^{\lambda} a_{k} z^{k}$

The convolution of the operators $\mathcal{R}_{q}^{\delta} f(z)$ and $I_{s, a, \mu}^{\lambda} f(z)$ defined as

$\mathcal{J}_{s, a, \mu}^{\delta, \lambda} f(z)=\mathcal{R}_{q}^{\delta} f(z) * I_{s, a, \mu}^{\lambda} f(z)=z+\sum_{k=2}^{\infty}\left(\frac{(k+\delta-1) !}{(\delta) !(k-1) !}\right)\left(\frac{1+a}{t+a}\right)^{s}\left(\frac{\mu}{\mu+k-1}\right)^{\lambda} a_{k} z^{k}$

$\mathcal{J}_{s, a, \mu}^{\delta, \lambda} f(z)=z+\sum_{k=2}^{\infty} \theta_{k, \delta} a_{k} z^{k}$

where $\theta_{k, \delta}=\left\{\left(\frac{(k+\delta-1) !}{(\delta) !(k-1) !}\right)\left(\frac{1+a}{t+a}\right)^{S}\left(\frac{\mu}{\mu+k-1}\right)^{\lambda}\right\}$. 
Now, for a function $f \in \mathcal{S}$, the function $h(z)=\left(f\left(z^{m}\right)\right)^{\frac{1}{m}} \quad(z \in \Theta, m \in \mathbb{N})$ is univalent. Further, that function maps $\Theta$ into an $m$-fold symmetry region. Thus, a function is named $m$ fold symmetric $([14,15])$ if it has the normalized form

$f(z)=z+\sum_{k=1}^{\infty} a_{m k+1} z^{m k+1}, \quad(z \in \Theta, m \in \mathbb{N})$.

Assume $\mathcal{S}_{m}$ is the class of $m$-fold symmetric univalent functions given by (3). We inform that the functions in $\mathcal{S}$ are one-fold symmetric. Similar to the definition of $m$-fold symmetric univalent functions, $m$-fold symmetric bi-univalent functions are presented. The series expansion of $f^{-1}$ is expressed by [16]

$$
\begin{aligned}
g(w)= & \left.w-a_{m+1} w^{m+1}+\left[(m+1) a_{m+1}^{2}-a_{2 m+1}\right)\right] w^{2 m+1}-\left[\frac{1}{2}(m+1)(3 m+2) a_{m+1}^{3}\right. \\
& \left.-(3 m+2) a_{m+1} a_{2 m+1}+a_{3 m+1}\right] w^{3 m+1}+\cdots,
\end{aligned}
$$

where $f^{-1}=g$. Assume $\sum_{m}$ is the class of $m$-fold symmetric bi-univalent functions in $\Theta$. If we set $m=1$, the formula (4) reduces to the formula (2) of the class $\sum$.

Next, Noonan and Thomas [17] introduced the $q^{\text {th }}$ Hankel determinant of $f$ by

$H_{q}(n)=\left|\begin{array}{lllc}a_{n} & a_{n+1} & \cdots & a_{n+q-1} \\ a_{n+1} & a_{n+2} & \cdots & a_{n+q} \\ \vdots & \vdots & \vdots & \vdots \\ a_{n+q-1} & a_{n+q} & \cdots & a_{n+2 q-2}\end{array}\right| \quad, \quad\left(a_{1}=1, n \geq 0, \quad q \geq 1\right)$.

Note that

$H_{2}(1)=\left|\begin{array}{ll}a_{1} & a_{2} \\ a_{2} & a_{3}\end{array}\right|=a_{3}-a_{2}^{2}, \quad H_{2}(2)=\left|\begin{array}{ll}a_{2} & a_{3} \\ a_{3} & a_{4}\end{array}\right|=a_{2} a_{4}-a_{3}^{2}$

and $H_{2}(1)$ is known as Fekete-Szegö functional (see [18]). After that, this approach has been studied by several researchers ([19-23]).

\section{MATERIAL METHOD}

To present our outcomes, we must recall some lemmas.

Lemma 2.1. [15] Assume $\mathcal{P}$ is class of functions $p$ analytic in $\Theta$ for which $\operatorname{Re}(p(z))>0$. If $p(z)=1+$ $p_{1} z+p_{2} z^{2}+\cdots$, then $\left|p_{i}\right| \leq 2$ for each $i \in \mathbb{N}$.

Lemma 2.2. [24] If $p \in \mathcal{P}$, then

$2 p_{2}=p_{1}^{2}+\left(4-p_{1}^{2}\right) x$

$4 p_{3}=p_{1}^{3}+2\left(4-p_{1}^{2}\right) p_{1} x-\left(4-p_{1}^{2}\right) p_{1} x^{2}+2\left(4-p_{1}^{2}\right)\left(1-|x|^{2}\right) z$

for some $x, z$ with $|x| \leq 1$ and $|z| \leq 1$.

Now, we shall introduce the class $\boldsymbol{\mathcal { C }}_{\Sigma \mathrm{m}}(\tau ; \beta)$ as follows.

Definition 3.1. A function $f \in \sum$ is in $\mathcal{C}_{\Sigma \mathrm{m}}(\tau ; \beta)(z, w \in \Theta, 0 \leq \tau \leq 1,0 \leq \beta<1, m \in \mathbb{N})$ if it fulfills 
$\operatorname{Re}\left[(2 \tau+1) \frac{z\left(\mathcal{J}_{s, a, \mu}^{\delta, \lambda} f(z)\right)^{\prime}}{z}-\tau z\left(\mathcal{J}_{s, a, \mu}^{\delta, \lambda} f(z)\right)^{\prime \prime}-2 \tau\right]>\beta$

and

$\operatorname{Re}\left[(2 \tau+1) \frac{w\left(J_{s, a, \mu}^{\delta, \lambda} g(w)\right)^{\prime}}{w}-\tau w\left(J_{s, a, \mu}^{\delta, \lambda} g(w)\right)^{\prime \prime}-2 \tau\right]>\beta$,

where the function $g$ is the extension of $f^{-1}$ to $\Theta$.

\section{THE RESEARCH FINDINGS AND DISCUSSION}

Theorem 3.1. Assume $f$ is in the class $\mathcal{C}_{\Sigma \mathrm{m}}(\tau ; \beta)$. For $0 \leq \tau \leq 1,0 \leq \beta<1$, we find $\left|a_{m+1} a_{3 m+1}-a_{2 m+1}^{2}\right|$

$\leq \begin{cases}E(\beta, 2-), & \text { if } \Phi(\beta, p) \geq 0 \text { and } \vartheta(\beta, p) \geq 0 \\ \frac{4(1-\beta)^{2}}{(2 m+1)^{2}[2 \tau(1-m)+1]^{2} \theta_{2 m+1, \delta}^{2},} & \text { if } \Phi(\beta, p) \leq 0 \text { and } \vartheta(\beta, p) \leq 0 \\ \max \left\{\frac{4(1-\beta)^{2}}{(2 m+1)^{2}[2 \tau(1-m)+1]^{2} \theta_{2 m+1, \delta}^{2}}, E(\beta, 2-)\right\}, & \text { if } \Phi(\beta, p)>0 \text { and } \vartheta(\beta, p)<0 \\ \max \left\{E\left(\beta, p_{0}\right), E(\beta, 2-)\right\}, & \text { if } \Phi(\beta, p)<0 \text { and } \vartheta(\beta, p)>0,\end{cases}$

where

$$
\begin{aligned}
& E(\beta, 2-)=\frac{4(1-\beta)^{2}}{(2 m+1)^{2}[2 \tau(1-m)+1]^{2} \theta_{2 m+1, \delta}^{2}}+(1-\beta)^{2}[8 \Phi(\beta, p)+2 \vartheta(\beta, p)], \\
& E\left(\beta, p_{0}\right)=\frac{4(1-\beta)^{2}}{(2 m+1)^{2}[2 \tau(1-m)+1]^{2} \theta_{2 m+1, \delta}^{2}}-\frac{(1-\beta)^{2} \vartheta^{2}(\beta, p)}{8 \Phi(\beta, p)}, \\
& p_{0}=\sqrt{\frac{-\vartheta(\beta, p)}{2 \Phi(\beta, p)},} \\
& \Phi(\beta, p)=\frac{(3 m+2)(1-\beta)}{2(m+1)^{2}[\tau(2-m)+1]^{4} \theta_{m+1, \delta}^{4}}-\frac{1}{2(m+1)^{2}(m+1)[\tau(2-m)+1]^{2}[2 \tau(1-m)+1]} \\
& \quad-\frac{1}{(m+1)(3 m+1)[\tau(2-3 m)+1][\tau(2-m)+1] \theta_{m+1, \delta} \theta_{3 m+1, \delta}}
\end{aligned}
$$

and

$$
\begin{aligned}
\vartheta(\beta, p)=\frac{2(3 m+2)(1-\beta)}{(m+1)^{2}(2 m+1)[\tau(2-m)+1]^{2}[2 \tau(1-m)+1]} \\
\quad+\frac{6}{(m+1)(3 m+1)[\tau(2-m)+1][\tau(2-3 m)+1] \theta_{m+1, \delta} \theta_{3 m+1, \delta}} \\
\quad-\frac{4}{(2 m+1)^{2}[2 \tau(1-m)+1]^{2} \theta_{2 m+1, \delta}^{2}} .
\end{aligned}
$$


Proof. Let $f \in \mathcal{C}_{\Sigma \mathrm{m}}(\tau ; \beta)$. Then

$$
\begin{aligned}
& (2 \tau+1) \frac{z\left(\mathcal{J}_{s, a, \mu}^{\delta, \lambda} f(z)\right)^{\prime}}{z}-\tau z\left(\mathcal{J}_{s, a, \mu}^{\delta, \lambda} f(z)\right)^{\prime \prime}-2 \tau=\beta+(1-\beta) p(z), \\
& (2 \tau+1) \frac{w\left(J_{s, a, \mu}^{\delta, \lambda} g(w)\right)^{\prime}}{w}-\tau w\left(\mathcal{J}_{s, a, \mu}^{\delta, \lambda} g(w)\right)^{\prime \prime}-2 \tau=\beta+(1-\beta) q(w),
\end{aligned}
$$

where $g=f^{-1}$ and

$$
\begin{aligned}
& p(z)=1+p_{m} z^{m}+p_{2 m} z^{2 m}+p_{3 m} z^{3 m}+\cdots, \\
& q(w)=1+q_{m} w^{m}+q_{2 m} w^{2 m}+q_{3 m} w^{3 m}+\cdots .
\end{aligned}
$$

If we equate the coefficients in (5) and (6), we find

$$
\begin{aligned}
& {[\tau(2-m)+1](m+1) \theta_{m+1, \delta} a_{m+1}=(1-\beta) p_{m},} \\
& {[2 \tau(1-m)+1](2 m+1) \theta_{2 m+1, \delta} a_{2 m+1}=(1-\beta) p_{2 m},} \\
& {[\tau(2-3 m)+1](3 m+1) \theta_{3 m+1, \delta} a_{3 m+1}=(1-\beta) p_{3 m},} \\
& -[\tau(2-m)+1](m+1) \theta_{m+1, \delta} a_{m+1}=(1-\beta) q_{m}, \\
& {[2 \tau(1-m)+1]\left[(m+1) a_{m+1}^{2}-a_{2 m+1}\right](2 m+1) \theta_{2 m+1, \delta}=(1-\beta) q_{2 m},} \\
& -[\tau(2-3 m)+1]\left[\frac{1}{2}(m+1)(3 m+2) a_{m+1}^{3}-(3 m+2) a_{m+1} a_{2 m+1}+a_{3 m+1}\right] \\
& (3 m+1) \theta_{3 m+1, \delta}=(1-\beta) q_{3 m} .
\end{aligned}
$$

From (7) and (10), we get

$$
\begin{aligned}
& p_{m}=-q_{m}, \\
& a_{m+1}=\frac{(1-\beta)}{(m+1)[\tau(2-m)+1] \theta_{m+1, \delta}} p_{m} .
\end{aligned}
$$

Subtracting (8) from (11), we have

$$
a_{2 m+1}=\frac{(1-\beta)^{2}}{2(m+1)[\tau(2-m)+1]^{2} \theta_{m+1, \delta}^{2}} p_{m}^{2}+\frac{(1-\beta)\left(p_{2 m}-q_{2 m}\right)}{2(2 m+1)[2 \tau(1-m)+1] \theta_{2 m+1, \delta}}
$$

Also, subtracting (9) from (12), we obtain

$$
\begin{aligned}
& a_{3 m+1}=\frac{(3 m+2)(1-\beta)^{2} p_{m}\left(p_{2 m}-q_{2 m}\right)}{4(m+1)(2 m+1)[\tau(2-m)+1][2 \tau(1-m)+1] \theta_{m+1, \delta} \theta_{2 m+1, \delta}} \\
& +\frac{(1-\beta)\left(p_{3 m}-q_{3 m}\right)}{2(3 m+1)[\tau(2-3 m)+1] \theta_{3 m+1, \delta}} .
\end{aligned}
$$

Then, we can establish that

$$
\left|a_{m+1} a_{3 m+1}-a_{2 m+1}^{2}\right|=\mid-\frac{(1-\beta)^{4}}{4(m+1)^{2}[\tau(2-m)+1]^{4} \theta_{m+1, \delta}^{4}} p_{m}^{4}
$$




$$
\begin{aligned}
& +\frac{(3 m+2)(1-\beta)^{3}}{4(m+1)^{2}(2 m+1)[\tau(2-m)+1]^{2}[2 \tau(1-m)+1] \theta_{m+1, \delta}} p_{m}^{2}\left(p_{2 m}-q_{2 m}\right) \\
& +\frac{(1-\beta)^{2}}{2(m+1)(3 m+1)[\tau(2-m)+1][\tau(2-3 m)+1] \theta_{m+1, \delta} \theta_{3 m+1, \delta}} p_{m}\left(p_{3 m}-q_{3 m}\right) \\
& -\frac{(1-\beta)^{2}}{4(2 m+1)^{2}[2 \tau(1-m)+1]^{2} \theta_{2 m+1, \delta}^{2}}\left(p_{2 m}-q_{2 m}\right)^{2} \mid
\end{aligned}
$$

According to Lemma (2.2) and in light of (13), we write

$$
\begin{aligned}
& \left.\begin{array}{l}
2 p_{2 m}=p_{m}^{2}+\left(4-p_{m}^{2}\right) x \\
2 q_{2 m}=q_{m}^{2}+\left(4-q_{m}^{2}\right) y
\end{array}\right\} \Rightarrow p_{2 m}-q_{2 m}=\frac{4-p_{m}^{2}}{2}(x-y), \\
& \begin{aligned}
4 p_{3 m}=p_{m}^{3} & +2\left(4-p_{m}^{2}\right) p_{m} x-p_{m}\left(4-p_{m}^{2}\right) x^{2}+2\left(4-p_{m}^{2}\right)\left(1-|x|^{2}\right) z, \\
4 q_{3 m}=q_{m}^{3} & +2\left(4-q_{m}^{2}\right) q_{m} y-q_{m}\left(4-q_{m}^{2}\right) y^{2}+2\left(4-q_{m}^{2}\right)\left(1-|y|^{2}\right) w, \\
p_{3 m}-q_{3 m} & =\frac{p_{m}^{3}}{2}+\frac{p_{m}\left(4-p_{m}^{2}\right)}{2}(x+y)-\frac{p_{m}\left(4-p_{m}^{2}\right)}{4}\left(x^{2}+y^{2}\right) \\
& +\frac{4-p_{m}^{2}}{2}\left[\left(1-|x|^{2}\right) z-\left(1-|y|^{2}\right) w\right] .
\end{aligned}
\end{aligned}
$$

Then, using (15) and (16) in (14), we get

$$
\begin{aligned}
& \left|a_{m+1} a_{3 m+1}-a_{2 m+1}^{2}\right|=\mid-\frac{(1-\beta)^{4}}{4(m+1)^{2}[\tau(2-m)+1]^{4} \theta_{m+1, \delta}^{4}} p_{m}^{4} \\
& +\frac{(3 m+2)(1-\beta)^{3}}{4(m+1)^{2}(2 m+1)[\tau(2-m)+1]^{2}[2 \tau(1-m)+1]} p_{m}^{2} \frac{\left(4-p_{m}^{2}\right)}{2}(x-y) \\
& +\frac{(1-\beta)^{2}}{2(m+1)(3 m+1)[\tau(2-m)+1][\tau(2-3 m)+1] \theta_{m+1, \delta} \theta_{3 m+1, \delta}} \frac{p_{m}^{4}}{2} \\
& +\frac{(1-\beta)^{2}}{2(m+1)(3 m+1)[\tau(2-m)+1][\tau(2-3 m)+1] \theta_{m+1, \delta} \theta_{3 m+1, \delta}} p_{m}^{2} \frac{4-p_{m}^{2}}{2}(x+y) \\
& -\frac{(1-\beta)^{2}}{2(m+1)(3 m+1)[\tau(2-m)+1][\tau(2-3 m)+1] \theta_{m+1, \delta} \theta_{3 m+1, \delta}} p_{m}^{2} \frac{\left(4-p_{m}^{2}\right)}{4}\left(x^{2}+y^{2}\right) \\
& +\frac{(1-\beta)^{2}}{2(m+1)(3 m+1)[\tau(2-m)+1][\tau(2-3 m)+1] \theta_{m+1, \delta} \theta_{3 m+1, \delta}} p_{m} \\
& \times \frac{\left(4-p_{m}^{2}\right)}{2}\left[\left(1-|x|^{2}\right) z-\left(1-|y|^{2}\right) w\right]-\frac{(1-\beta)^{2}}{4(2 m+1)^{2}[2 \tau(1-m)+1]^{2} \theta_{2 m+1, \delta}^{2}} \\
& \times \frac{\left(4-p_{m}^{2}\right)^{2}}{4}(x-y)^{2} \mid . \\
& \left|a_{m+1} a_{3 m+1}-a_{2 m+1}^{2}\right| \leq \frac{(1-\beta)^{4}}{4(m+1)^{2}[\tau(2-m)+1]^{4} \theta_{m+1, \delta}^{4}} p_{m}^{4} \\
& +\frac{(1-\beta)^{2}}{2(m+1)(3 m+1)[\tau(2-m)+1][\tau(2-3 m)+1] \theta_{m+1, \delta} \theta_{3 m+1, \delta}} \frac{(1-\beta)^{2}}{2} \\
& +\frac{(3 m+2)(1-\beta)^{3}}{2(m+1)(3 m+1)[\tau(2-m)+1][\tau(2-3 m)+1] \theta_{m+1, \delta} \theta_{3 m+1, \delta}} p_{m}\left(4-p_{m}^{2}\right) \\
& +(|x|+|y|)\left[\frac{(1-\beta)^{2}}{4(m+1)^{2}(2 m+1)[\tau(2-m)+1]^{2}[2 \tau(1-m)+1]} p_{m}^{2} \frac{\left(4-p_{m}^{2}\right)}{2}\right.
\end{aligned}
$$




$$
\begin{aligned}
& +\left(|x|^{2}+|y|^{2}\right)\left[\frac{(1-\beta)^{2}}{2(m+1)(3 m+1)[\tau(2-m)+1][\tau(2-3 m)+1] \theta_{m+1, \delta} \theta_{3 m+1, \delta}} p_{m}^{2} \frac{\left(4-p_{m}^{2}\right)}{4}\right. \\
& \left.-\frac{(1-\beta)^{2}}{2(m+1)(3 m+1)[\tau(2-m)+1][\tau(2-3 m)+1] \theta_{m+1, \delta} \theta_{3 m+1, \delta}} p_{m} \frac{\left(4-p_{m}^{2}\right)}{4}\right] \\
& +\frac{(1-\beta)^{2}}{4(2 m+1)^{2}[2 \tau(1-m)+1]^{2} \theta_{2 m+1, \delta}^{2}} \frac{\left(4-p_{m}^{2}\right)^{2}}{4}(|x|+|y|)^{2} .
\end{aligned}
$$

Since $p \in P,\left|p_{m}\right| \leq 2$. Letting $\left|p_{m}\right|=p$, we may assume without restriction that $p \in[0,2]$. For $\gamma=|x| \leq$ 1 and $\alpha=|y| \leq 1$, we get

$\left|a_{m+1} a_{3 m+1}-a_{2 m+1}^{2}\right| \leq A_{1}+(\gamma+\alpha) A_{2}+\left(\gamma^{2}+\alpha^{2}\right) A_{3}+(\gamma+\alpha)^{2} A_{4}=H(\gamma, \alpha)$

where

$$
\begin{aligned}
A_{1}=A_{1}(\beta, p)= & \frac{(1-\beta)^{2}}{2(m+1)[\tau(2-m)+1] \theta_{m+1, \delta}}\left[\left(\frac{1}{2(m+1)[\tau(2-m)+1]^{3} \theta_{m+1, \delta}^{3}}\right.\right. \\
& \left.+\frac{1}{2(3 m+1)[\tau(2-3 m)+1] \theta_{3 m+1, \delta}}\right) p^{4}-\frac{(1-\beta)^{2}}{(3 m+1)[\tau(2-3 m)+1] \theta_{3 m+1, \delta}} p^{3} \\
& \left.+\frac{4}{(3 m+1)[\tau(2-3 m)+1] \theta_{3 m+1, \delta}} p\right] \geq 0 \\
A_{2}=A_{2}(\beta, p)= & \frac{(1-\beta)^{2}}{4(m+1)[\tau(2-m)+1]} p^{2}\left(4-p^{2}\right) \\
A_{3}=A_{3}(\beta, p)= & \left.\frac{\left[\frac{(3 m+2)(1-\beta)}{2(m+1)(2 m+1)[\tau(2-m)+1][2 \tau(1-m)+1]}\right.}{8(3 m+1)[\tau(2-3 m)+1] \theta_{m+1, \delta} \theta_{3 m+1, \delta}}\right] \geq 0, \\
A_{4}=A_{4}(\beta, p)= & \frac{(3 m+1)[\tau(2-m)+1][\tau(2-3 m)+1] \theta_{m+1, \delta} \theta_{3 m+1, \delta}}{4(2 m+1)^{2}[2 \tau(1-m)+1]^{2} \theta_{2 m+1, \delta}^{2}}
\end{aligned}
$$

Now, we aim to maximize $H(\gamma, \alpha)$ on the square $[0,1] \times[0,1]$. Hence, we need the maximum of $H(\gamma, \alpha)$ for the cases $p \in(0,2), p=0$ and $p=2$. If $p \in(0,2)$, we find $A_{3}<0$ and $A_{3}+2 A_{4}>0$. Thus, we conclude that

$H_{\gamma \gamma} \cdot H_{\alpha \alpha}-\left(H_{\gamma \alpha}\right)^{2}<0$

which means that $H$ cannot have a local maximum in the interior of the square. Hence, we examine the boundary of the square.

For $\gamma=0$ and $0 \leq \alpha \leq 1$ (similarly $\alpha=0$ and $0 \leq \gamma \leq 1$ ), we have

$H(0, \alpha)=N(\alpha)=\left(A_{3}+A_{4}\right) \alpha^{2}+A_{2} \alpha+A_{1}$. 
1- The case $A_{3}+A_{4} \geq 0$ : For $0<\alpha<1$ and any fixed $p(0<p<2)$, we find that $N^{\prime}(\alpha)=$ $2\left(A_{3}+A_{4}\right) \alpha+A_{2}>0$, that is, $N(\alpha)$ is increasing. Thus, the maximum of $N(\alpha)$ occurs at $\alpha=1$ and $\max N(\alpha)=N(1)=A_{1}+A_{2}+A_{3}+A_{4}$.

2- The case $A_{3}+A_{4}<0$ : Since $A_{2}+2\left(A_{3}+A_{4}\right) \geq 0$, we find that $A_{2}+2\left(A_{3}+A_{4}\right)<2\left(A_{3}+A_{4}\right) \alpha+$ $A_{2}<A_{2}$ and so $N^{\prime}(\alpha)>0$. Thus, the maximum of $N(\alpha)$ occurs at $\alpha=1$.

If $p=2$, we get

$$
\begin{aligned}
& H(\gamma, \alpha)=\frac{(1-\beta)^{2}}{(m+1)[\tau(2-m)+1] \theta_{m+1, \delta}} \\
& \times\left[\frac{4(1-\beta)^{2}}{(m+1)[\tau(2-m)+1]^{3} \theta_{m+1, \delta}^{3}}+\frac{4}{(3 m+1)[\tau(2-3 m)+1] \theta_{3 m+1, \delta}}\right] .
\end{aligned}
$$

From (18) and the above cases, we arrive

$$
\max N(\alpha)=N(1)=A_{1}+A_{2}+A_{3}+A_{4} \quad(0 \leq \alpha \leq 1,0 \leq p \leq 2) .
$$

For $\gamma=1$ and $0 \leq \alpha \leq 1$ (similarly $\alpha=1$ and $0 \leq \gamma \leq 1$ ), we obtain

$$
H(1, \alpha)=L(\alpha)=\left(A_{3}+A_{4}\right) \alpha^{2}+\left(A_{2}+2 A_{4}\right) \alpha+A_{1}+A_{2}+A_{3}+A_{4} .
$$

Similar to the above cases of $A_{3}+A_{4}$, we have

$\max L(\alpha)=L(1)=A_{1}+2 A_{2}+2 A_{3}+4 A_{4}$.

Since $N(1) \leq L(1)$ for $p \in[0,2], \max H(\gamma, \alpha)=H(1,1)$. Therefore, the maximum of $H$ occurs at $\gamma=$ 1 and $\alpha=1$ in the closed square.

Let $E:[0,2] \rightarrow R$

$$
E(\beta, p)=\max H(\gamma, \alpha)=H(1,1)=A_{1}+2 A_{2}+2 A_{3}+4 A_{4} .
$$

Substituting the values of $A_{1}, A_{2}, A_{3}$ and $A_{4}$ from (19) yields

$$
\begin{aligned}
E(\beta, p)=\frac{(1-\beta)^{2}}{2} & {\left[\left(\frac{(1-\beta)^{2}}{2(m+1)^{2}[\tau(2-m)+1]^{4} \theta_{m+1, \delta}^{4}}\right.\right.} \\
& -\frac{(3 m+2)(1-\beta)}{2(m+1)^{2}(2 m+1)[\tau(2-m)+1]^{2}[2 \tau(1-m)+1]} \\
& -\frac{1}{(m+1)(3 m+1)[\tau(2-3 m)+1][\tau(2-m)+1] \theta_{m+1, \delta} \theta_{3 m+1, \delta}} \\
& \left.+\frac{1}{2(2 m+1)^{2}[2 \tau(1-m)+1]^{2} \theta_{2 m+1, \delta}^{2}}\right) p^{4} \\
& +\left(\frac{2(3 m+2)(1-\beta)}{(m+1)^{2}(2 m+1)[\tau(2-m)+1]^{2}[2 \tau(1-m)+1]}\right. \\
& +\frac{6}{(m+1)(3 m+1)[\tau(2-m)+1][\tau(2-3 m)+1] \theta_{m+1, \delta} \theta_{3 m+1, \delta}} \\
& \left.\left.-\frac{4}{(2 m+1)^{2}[2 \tau(1-m)+1]^{2} \theta_{2 m+1, \delta}^{2}}\right) p^{2}+\frac{8}{(2 m+1)^{2}[2 \tau(1-m)+1]^{2} \theta_{2 m+1, \delta}^{2}}\right]
\end{aligned}
$$




$$
E(\beta, p)=\frac{(1-\beta)^{2}}{2}\left[\Phi(\beta, p) p^{4}+\vartheta(\beta, p) p^{2}\right]+\frac{4(1-\beta)^{2}}{(2 m+1)^{2}[2 \tau(1-m)+1]^{2} \theta_{2 m+1, \delta}^{2}}
$$

where

$$
\begin{aligned}
& \Phi(\beta, p)=\frac{(1-\beta)^{2}}{2(m+1)^{2}[\tau(2-m)+1]^{4} \theta_{m+1, \delta}^{4}}-\frac{(3 m+2)(1-\beta)}{2(m+1)^{2}(m+1)[\tau(2-m)+1]^{2}[2 \tau(1-m)+1]} \\
& \quad-\frac{1}{(m+1)(3 m+1)[\tau(2-3 m)+1][\tau(2-m)+1] \theta_{m+1, \delta} \theta_{3 m+1, \delta}} \\
& \quad+\frac{1}{2(2 m+1)^{2}[2 \tau(1-m)+1]^{2} \theta_{2 m+1, \delta}^{2}}, \\
& \vartheta(\beta, p)=\frac{2(3 m+2)(1-\beta)}{(m+1)^{2}(2 m+1)[\tau(2-m)+1]^{2}[2 \tau(1-m)+1]} \\
& \quad+\frac{6}{(m+1)(3 m+1)[\tau(2-m)+1][\tau(2-3 m)+1] \theta_{m+1, \delta} \theta_{3 m+1, \delta}} \\
& \quad-\frac{4}{(2 m+1)^{2}[2 \tau(1-m)+1]^{2} \theta_{2 m+1, \delta}^{2}} .
\end{aligned}
$$

Assume that $E(\beta, p)$ has a maximum value in an interior of $p \in[0,2]$, then

$E^{\prime}(\beta, p)=(1-\beta)^{2}\left[2 \Phi(\beta, p) p^{3}+\vartheta(\beta, p) p\right]$

Now, we msut investigate the function $E^{\prime}(\beta, p)$ due to the different cases of $\Phi(\beta, p)$ and $\vartheta(\beta, p)$.

(i) Let $\Phi(\beta, p) \geq 0$ and $\vartheta(\beta, p) \geq 0$, then $E^{\prime}(\beta, p) \geq 0$, so $E(\beta, p)$ is an increasing function. Therefore,

$$
\begin{aligned}
\max \{E(\beta, p): p \in(0,2)\}=E(\beta, 2-) & =\frac{4(1-\beta)^{2}}{(2 m+1)^{2}[2 \tau(1-m)+1]^{2} \theta_{2 m+1, \delta}^{2}} \\
& +(1-\beta)^{2}[8 \Phi(\beta, p)+2 \vartheta(\beta, p)],
\end{aligned}
$$

that is,

$\max \{\max \{H(\gamma, \alpha): \gamma, \alpha \in[0,1]\}: p \in(0,2)\}=E(\beta, 2-)$

(ii) Let $\Phi(\beta, p) \leq 0$ and $\vartheta(\beta, p) \leq 0$, then $E^{\prime}(\beta, p) \leq 0$. Thus, $E(\beta, p)$ is a decreasing. Therefore,

$$
\max \{E(\beta, p): p \in(0,2)\}=E(\beta, 0+)=\frac{4(1-\beta)^{2}}{(2 m+1)^{2}[2 \tau(1-m)+1]^{2} \theta_{2 m+1, \delta}^{2}} .
$$

(iii) Let $\Phi(\beta, p)>0$ and $\vartheta(\beta, p)<0$, and then $p_{0}=\sqrt{\frac{-\vartheta(\beta, p)}{2 \Phi(\beta, p)}}$ is a critical point. Let $p_{0} \in(0,2)$. Since $E^{\prime \prime}\left(\beta, p_{0}\right)>0, p_{0}$ is local minimum point of $E(\beta, p)$. Therefore, $E(\beta, p)$ cannot have a local maximum.

(iv) If $\Phi(\beta, p)<0$ and $\vartheta(\beta, p)>0$, then $p_{0}$ is critical point of $E(\beta, p)$. Since $E^{\prime \prime}\left(\beta, p_{0}\right)<0, p_{0}$ is local maximum point for $p_{0} \in(0,2)$ and so the maximum value occurs at $p=p_{0}$. Hence,

$\max \{E(\beta, p): p \in(0,2)\}=E\left(\beta, p_{0}\right)$,

where 
$E\left(\beta, p_{0}\right)=\frac{4(1-\beta)^{2}}{(2 m+1)^{2}[2 \tau(1-m)+1]^{2} \theta_{2 m+1, \delta}^{2}}-\frac{(1-\beta)^{2} \vartheta^{2}(\beta, p)}{8 \Phi(\beta, p)}$

Remark 3.1. For $\tau=0$, a function $f \in \sum$ belongs to the class $\mathcal{C}_{\Sigma \mathrm{m}}(\tau ; \beta)$ if it fullfils

$\operatorname{Re}\left[\frac{z\left(\mathcal{J}_{s, a, \mu}^{\delta, \lambda} f(z)\right)^{\prime}}{z}\right]^{\prime}>\beta$,
$\operatorname{Re}\left[\frac{w\left(\mathcal{J}_{s, a, \mu}^{\delta, \lambda} g(w)\right)^{\prime}}{w}\right]>\beta$.

If we let $\tau=0$, we arrive at Corollary 3.1.

Corollary 3.1. Let $f$ given by (1) belong to the class $\boldsymbol{C}_{\Sigma \mathrm{m}}(0 ; \beta)=\mathfrak{D}_{\Sigma \mathrm{m}}(\beta)$. Then

$$
\left|a_{m+1} a_{3 m+1}-a_{2 m+1}^{2}\right| \leq\left\{\begin{array}{ll}
E(\beta, 2-), & \text { if } \Phi(\beta, p) \geq 0 \text { and } \vartheta(\beta, p) \geq 0 \\
\frac{4(1-\beta)^{2}}{(2 m+1)^{2} \theta_{2 m+1, \delta}^{2}}, & \text { if } \Phi(\beta, p) \leq 0 \text { and } \vartheta(\beta, p) \leq 0 \\
\max \left\{\frac{4(1-\beta)^{2}}{(2 m+1)^{2} \theta_{2 m+1, \delta}^{2}}, E(\beta, 2-)\right\}, & \text { if } \Phi(\beta, p)>0 \text { and } \vartheta(\beta, p)<0 \\
\max \left\{E\left(\beta, p_{0}\right), E(\beta, 2-)\right\}, & \text { if } \Phi(\beta, p)<0 \text { and } \vartheta(\beta, p)>0
\end{array},\right.
$$

where

$$
\begin{aligned}
& E(\beta, 2-)=\frac{4(1-\beta)^{2}}{(2 m+1)^{2} \theta_{2 m+1, \delta}^{2}}+(1-\beta)^{2}[8 \Phi(\beta, p)+2 \vartheta(\beta, p)] \\
& E\left(\beta, p_{0}\right)=\frac{4(1-\beta)^{2}}{(2 m+1)^{2} \theta_{2 m+1, \delta}^{2}}-\frac{(1-\beta)^{2} \vartheta^{2}(\beta, p)}{8 \Phi(\beta, p)}, \quad p_{0}=\sqrt{\frac{-\vartheta(\beta, p)}{2 \Phi(\beta, p)}} \\
& \Phi(\beta, p)=\frac{(1-\beta)^{2}}{2(m+1)^{2} \theta_{m+1, \delta}^{4}}-\frac{(3 m+2)(1-\beta)}{2(m+1)^{2}(m+1)}-\frac{1}{(m+1)(3 m+1) \theta_{m+1, \delta} \theta_{3 m+1, \delta}} \\
& \quad+\frac{1}{2(2 m+1)^{2} \theta_{2 m+1, \delta}^{2}}, \\
& \vartheta(\beta, p)=\frac{2(3 m+2)(1-\beta)}{(m+1)^{2}(2 m+1)}+\frac{6}{(m+1)(3 m+1) \theta_{m+1, \delta} \theta_{3 m+1, \delta}}-\frac{4}{(2 m+1)^{2} \theta_{2 m+1, \delta}^{2}}
\end{aligned}
$$

\section{CONCLUSION}

In the study carried out, a new operator was described via convolution. By using this operator, a new class was presented and studied.

For the future studies, it is planned to describe new subclasses of $m$-fold symmetric bi-univalent functions. It is also planned to present upper bounds for initial Taylor coefficients, Fekete-Szegö and Hankel determinant inequalities for functions in the defined classes. 


\section{CONFLICTS OF INTEREST}

No conflict of interest was declared by the authors.

\section{REFERENCES}

[1] Duren, P. L., “Univalent Functions”, Springer- Verlag, New York, (1983).

[2] Srivastava, H. M., Mishra, A. K., Gochhayat, P., "Certain subclasses of analytic and bi-univalent functions", Applied Mathematics Letters, 23(10): 1188-1192, (2010).

[3] Atshan, W. G., Yalçın, S., Hadi, R. A., "Coefficient estimates for special subclasses of $k$-fold symmetric bi-univalent functions", Mathematics for Applications, 9: 83-90, (2020).

[4] Brannan, D. A., Clunie, J. G., "Aspects of contemporary complex analysis", Proceedings of the NATO Advanced Study Institute Held at University of Durham, New York: Academic Press, (1979).

[5] Brannan, D. A., Taha, T. S., "On some classes of bi-univalent functions", Studia Universitatis Babeş-Bolyai Mathematica, 31(2): 70-77, (1986).

[6] Çağlar, M., Deniz, E., "Initial coefficients for a subclass of bi-univalent functions defined by Salagean differential operator", Communications Faculty of Sciences University of Ankara Series A1 Mathematics and Statistics, 66 (1): 85-91, (2017).

[7] Kazımoğlu, S., Deniz, E., "Fekete-Szegö problem for generalized bi-subordinate functions of complex order", Hacettepe Journal of Mathematics and Statistics, 49(5): 1695-1705, (2020).

[8] Lewin, M., "On a coefficient problem for bi-univalent functions", Proceding of the American Mathematical Society, 18: 63-68, (1967).

[9] Netanyahau, E., "The minimal distance of the image boundary from the origin and the second coefficient of a univalent function in $|\mathrm{z}|<1$ ", Archive for Rotional Mechanic and Analysis, 32(2): 100-112, (1969).

[10] Yalçın, S., Atshan, W. G., Hassan, H. Z., "Coefficients assessment for certain subclasses of biunivalent functions related with quasi-subordination", Publications De L' Institut Mathematique, Nouvelle seŕie, 108(122): 155-162, (2020).

[11] Aldweby, H., Darus, M., "Some subordination results on $q$-analogue of Ruscheweyh differential operator", Abstract and Applied Analysis, 2014: 1-6, (2014).

[12] Komatu, Y., "On analytic prolongation of family of integral operators", Mathematica (Cluj), 32(55): 141-145, (1990).

[13] Atshan, W. G., Badawi, E. I., "On sandwich theorems for certain univalent function defined by a new operator", Journal of Al-Qadisiyah for Computer Science and Mathematics, 11(2): 72-80, (2019).

[14] Koepf, W., "Coefficient of symmetric functions of bounded boundary rotations", Proceding of the American Mathematical Society, 105: 324-329, (1989).

[15] Pommerenke, Ch., "Univalent Functions", Vandenhoeck and Ruprecht, Gottingen, (1975). 
[16] Srivastava, H. M., Gaboury, S., Ghanim, F., "Coefficient estimates for some subclasses of $m$-fold symmetric bi-univalent functions", Acta Universitatis Apulensis, 41: 153-164, (2015).

[17] Noonan, J. W., Thomas, D. K., "On the second Hankel determinant of areally mean $p$-valent functions", Transactions of the American Mathematical Society, 223(2): 337-346, (1976).

[18] Fekete, M., Szegö, G., "Eine bemerkung uber ungerade schlichte funktionen”, Journal of London Mathematical Society, 2: 85-89, (1933).

[19] Altınkaya, Ş., Yalçın, S., "Second Hankel determinant for a general subclass of bi-univalent functions", TWMS Journal of Pure and Applied Mathematics, 7(1): 98-104, (2015).

[20] Çağlar, M., Deniz, E., Srivastava, H. M., "Second Hankel determinant for certain subclasses of biunivalent functions", Turkish Journal of Mathematics, 41 (3): 694-706, (2017).

[21] Deniz, E., Çağlar, M., Orhan, H., "Second hankel determinant for bi-starlike and biconvex functions of order $\beta$ ", Applied Mathematics and Computation, 271: 301-307, (2015).

[22] Hayami, T., Owa, S., "Generalized Hankel determinant for certain classes", International Journal of Mathematical Analysis, 52(4): 2473-2585, (2010).

[23] Yavuz, T., "Second Hankel determinant for analytic functions defined by Ruscheweyh derivative", International Journal of Analysis and Applications, 8(1): 63-68, (2015).

[24] Grenander, U., Szegö, G., "Toeplitz forms and their applications", California Monographs in Mathematical Sciences, University of California Press, Berkeley, (1958). 